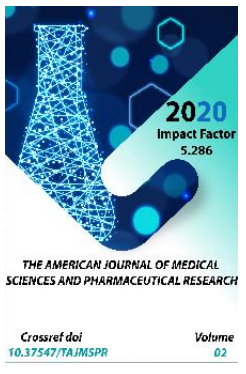

\title{
Commonness And Danger Variables Of Smoking Among Secondary School Female Understudies In Arar
}

\author{
Abdulaziz Alfayez Nourah \\ Family And Community Medicine Dept., Alfaisal University, Saudi Arabia
}

\section{ABSTRACT}

Smoking is the main worldwide reason for preventable passings. Studies have demonstrated that smoking commencement during youth improves the probability of kept smoking during adulthood and diminishes the opportunity of stopping. Numerous past explores have been directed in various districts of Saudi Arabia to recognize the pervasiveness of smoking among guys; barely any examinations remembered females for various territory however no investigations have been led among female at northern line of KSA. This examination meant to deciding the commonness of smoking and distinguish hazard factors among secondary school female understudies in Arar, KSA 2018.

\section{KEYWORDS}

Smoking pervasiveness, female, young lady, secondary school understudies, Arar.

\section{INTRODUCTION}

Tobacco smoking is one of the greatest general medical issues on the planet with in excess of 6 million passings every year as aftereffect of direct tobacco use and around 890000 passings per year as consequence of nonsmokers being presented to recycled smoke. 
WHO appraises that $80 \%$ of the world's 1.1 billion smokers live in low-and center pay nations . Nonetheless, in non-industrial nations, tobacco utilization keeps on expanding by around 3.4\% every year. Tobacco use is the main worldwide reason for preventable Deaths. Similar to the essential danger factor of unexpected passing in guys and second in females among average age.

The commonness of smoking contrasted from area to another as they found that Aljwaf, Northen Borders areas have the most elevated predominance while the least pervasiveness was found from grown-ups who live in Aseer Jizan and Albaha. In Hail, an examination by Algorinees et al. To evaluate the pervasiveness of smoking among the male optional school understudies found that $19.5 \%$ of understudies were current smokers. In that review, 79\% of the smokers revealed that they began smoking since they imitated the grown-up. Also $27 \%$ of smoker members revealed that their families realize that they smoke.

\section{Information Assortment Device}

Self-controlled poll was utilized in the examination was in Arabic language under administrator creator in missing of instructors to keep up private. The survey included 18 Variables from which is a Global Standard School-Based for deliberately checking tobacco use among youth and following key tobacco control pointers, It incorporates 56 inquiries planned, certain inquiries chose to cover the goal of this examination.

Thus, in this examination, we meant to recognize the commonness of smoking among the female secondary school understudies in Arar matured 14 to 17 years and it was discovered that $19.2 \%$ of the members were smokers. The discoveries of our examination are in close concurrences with results from beforehand considers which announced that in Hail the pervasiveness of smoking was $19.5 \%$. In spite of the fact that the investigation was done uniquely among guys, likenesses can be drawn between the two urban areas both situated in the north and offer comparative social, and financial variables .

It is as yet considered very conceivable that the pervasiveness of smoking when contrasted and different examinations was done in different areas of Saudi Arabia among juvenile and this might be because of various sexual orientation this may assume principle job in the distinctive between result add to this pressing factor from social, culture and strict factor and as we probably are aware Saudi is traditionalist society. Our outcomes propose the most grounded factor to start smoking was identified with companions and a few family members smokers at home other than guardians. The companion pressure was additionally distinguished to contribute fundamentally in settling on the choice of being a smoker which is predictable with different investigations, for example, that completed in Jeddah which proposed that one of the impacting factor is level of schooling of guardians . Furthermore, as in National Guard region in Riyadh proposing that one of the principle factor is having available time and alleviation from stress. Moreover, over portion of the smoker members endeavored to stop smoking if post-smoking help was concurred to them suitably. There were a few restrictions anyway in our investigation. Our review was distinctly among female understudies, this may in future urge doctors to do concentrates among male understudies too. 


\section{CONCLUSION}

We need to expand level of Awareness just as, smoking suspension programs coordinated the young people are critically required in this district. As in this investigation show the main age to begin smoking is at $16-17$ years and more seasoned a large portion of them on evaluation 11 , so it should provoke the wellbeing training to begin informing about the destructive impacts with respect to smoking in middle school classes.

\section{REFERENCES}

1. Sex contrasts in cardiovascular danger components and infection anticipation Science Direct. Accessible from:Gotten to on 9 July 2018.

2. Algabbani $A M$, Almubark $R$, Althumiri $N$, Alqahtani A, BinDhim N. The Prevalence of Cigarette Smoking in Saudi Arabia in 2017; 1(1):1-1. Accessible from:Gotten to on 9 July 2017.

3. Rakan Mosa Algorinees et al. Pervasiveness of Cigarette Smoking Usage among Adolescent Students in Northern Saudi Arabia. Asian Pacific Journal of Cancer Prevention. 2015; 14(4):1437-1441. Gotten to on 9 July 2015. 